\title{
Existence of positive solutions of elliptic mixed boundary value problem
}

\section{Guofa Li*}

${ }^{*}$ Correspondence:

ligf8855@yahoo.com.cn

Department of Mathematics and

Information Science, Qujing Normal

University, Qujing, 655011, P.R. China

\section{Abstract}

In this paper, we use variational methods to prove two existence of positive solutions of the following mixed boundary value problem:

$$
\begin{cases}-\Delta u=f(x, u), & x \in \Omega, \\ u=0, & x \in \sigma, \\ \frac{\partial u}{\partial v}=g(x, u), & x \in \Gamma .\end{cases}
$$

One deals with the asymptotic behaviors of $f(x, u)$ near zero and infinity and the other deals with superlinear of $f(x, u)$ at infinity.

MSC: 35M12;35D30

Keywords: elliptic mixed boundary value problem; positive solutions; mountain pass theorem; Sobolev embedding theorem

\section{Introduction and preliminaries}

This paper is concerned with the existence of positive solutions of the following elliptic mixed boundary value problem:

$$
\begin{cases}-\Delta u=f(x, u), & x \in \Omega, \\ u=0, & x \in \sigma, \\ \frac{\partial u}{\partial v}=g(x, u), & x \in \Gamma,\end{cases}
$$

where $\Omega$ is a bounded domain in $\mathbb{R}^{n}$ with Lipschitz boundary $\partial \Omega, \sigma \cup \Gamma=\partial \Omega, \sigma \cap \Gamma=\emptyset, \Gamma$ is a sufficiently smooth $(n-1)$-dimensional manifold, and $v$ is the outward normal vector on $\partial \Omega$. We assume $f: \Omega \times \mathbb{R} \rightarrow \mathbb{R}, g: \Gamma \times \mathbb{R} \rightarrow \mathbb{R}$ are continuous and satisfy

(S1) $f(x, t) \geq 0, \forall t \geq 0, x \in \Omega, f(x, 0)=0 . f(x, t) \equiv 0, \forall t<0, x \in \Omega$.

(S2) For almost every $x \in \Omega, \frac{f(x, t)}{t}$ is nondecreasing with respect to $t>0$.

(S3) $\lim _{t \rightarrow 0} \frac{f(x, t)}{t}=p(x), \lim _{t \rightarrow+\infty} \frac{f(x, t)}{t}=q(x) \not \equiv 0$ uniformly in a.e. $x \in \Omega$, where $\|p(x)\|_{\infty}<\lambda_{1}, \lambda_{1}$ is the first eigenvalue of (2), $0 \leq p(x), q(x) \in L^{\infty}(\Omega)$.

(S4) There exists $c_{1}, c_{2}>0$ such that $|f(x, t)| \leq c_{1}+c_{2}|t|^{p-1}$ for some $p \in\left(2, \frac{2 n}{n-2}\right)$ as $n \geq 3$ and $p \in(2,+\infty)$ as $n=1,2$. 
The eigenvalue problem of (1) is studied by Liu and Su in [1]

$$
\begin{cases}-\Delta u=\lambda u & \text { in } \Omega \\ u=0 & \text { on } \sigma \\ \frac{\partial u}{\partial v}=\lambda u & \text { on } \Gamma\end{cases}
$$

There exists a set of eigenvalues $\left\{\lambda_{k}\right\}$ and corresponding eigenfunctions $\left\{u_{k}\right\}$ which solve problem (2), where $0 \leq \lambda_{1} \leq \lambda_{2} \leq \cdots \leq \lambda_{k} \leq \cdots, \lambda_{k} \rightarrow \infty$ as $k \rightarrow \infty, \lambda_{1}=$ $\inf _{0 \neq u \in V} \frac{\int_{\Omega}|\nabla u|^{2} d x}{\int_{\Omega}|u|^{2} d x+\int_{\Gamma}|u|^{2} d s}$.

There have been many papers concerned with similar problems at resonance under the boundary condition; see [2-10]. Moreover, some multiplicity theorems are obtained by the topological degree technique and variational methods; interested readers can see [11-17]. Problem (1) is different from the classical ones, such as those with Dirichlet, Neuman, Robin, No-flux, or Steklov boundary conditions.

In this paper, we assume $V:=\left\{v \in H^{1}(\Omega):\left.v\right|_{\sigma}=0\right\}$ is a closed subspace of $H^{1}(\Omega)$. We define the norm in $V$ as $\|u\|^{2}=\int_{\Omega}|\nabla u|^{2} d x+\int_{\Gamma}|\gamma u|^{2} d s,\|\cdot\|_{L^{p}(\Omega)}$ is the $L^{p}(\Omega)$ norm, $\|\cdot\|_{L^{p}(\Gamma)}$ is the $L^{p}(\Gamma)$ norm, $\gamma: V \rightarrow L^{2}(\Gamma)$ is the trace operator with $\gamma u=u_{\Gamma}$ for all $u \in H^{1}(\Omega)$, that is continuous and compact (see [18]). Furthermore, we define $g=\gamma f$, $0 \leq g(x, t) \leq|\gamma f(x, t)|$ for $t>0$ (see [1]). Then, by (S3), we obtain

$$
\lim _{t \rightarrow+\infty} \frac{g(x, t)}{t} \leq \lim _{t \rightarrow+\infty} \frac{|\gamma f(x, t)|}{t}=q(x) \not \equiv 0, \quad \text { a.e. } x \in \bar{\Omega} .
$$

Let $\Omega$ be a bounded domain with a Lipschitz boundary; there is a continuous embedding $V \hookrightarrow L^{y}(\Omega)$ for $y \in\left[2, \frac{2 n}{n-2}\right]$ when $n \geq 3$, and $y \in[2,+\infty)$ when $n=1,2$. Then there exists $\gamma_{y}>0$, such that

$$
\|u\|_{L^{y}(\Omega)} \leq \gamma_{y}\|u\|, \quad \forall u \in V .
$$

Moreover, there is a continuous boundary trace embedding $V \hookrightarrow L^{z}(\Gamma)$ for $z \in\left[2, \frac{2(n-1)}{n-2}\right]$ when $n \geq 3$, and $z \in[2,+\infty)$ when $n=1,2$. Then there exists $k_{z}>0$, such that

$$
\|u\|_{L^{z}(\Gamma)} \leq k_{z}\|u\|, \quad \forall u \in V .
$$

It is well known that to seek a nontrivial weak solution of problem (1) is equivalent to finding a nonzero critical value of the $C^{1}$ functional

$$
J(u)=\frac{1}{2} \int_{\Omega}|\nabla u|^{2} d x-\int_{\Omega} F(x, u) d x-\int_{\Gamma} G(s, u) d s,
$$

where $u \in V, F(x, u)=\int_{0}^{u} f(x, t) d t, G(x, u)=\int_{0}^{u} g(x, t) d t$. Moreover, by (S1) and the Strong maximum principle, a nonzero critical point of $J$ is in fact a positive solution of (1). In order to find critical points of the functional (6), one often requires the technique condition, that is, for some $\mu>2, \forall|u| \geq M>0, x \in \Omega$,

$$
0<\mu F(x, u) \leq u f(x, u), \quad F(x, u)=\int_{0}^{u} f(x, t) d t .
$$


It is easy to see that the condition (AR) implies that $\lim _{u \rightarrow+\infty} \frac{F(x, u)}{u^{2}}=+\infty$, that is, $f(x, u)$ must be superlinear with respect to $u$ at infinity. In the present paper, motivated by [19] and [20], we study the existence and nonexistence of positive solutions for problem (1) with the asymptotic behavior assumptions (S3) of $f$ at zero and infinity. Moreover, we also study superlinear of $f$ at infinity with $q(x) \equiv+\infty$ in (S3), which is weaker than the (AR) condition, that is the (AR) condition does not hold.

In order to get our conclusion, we define the minimization problem

$$
\Lambda=\inf \left\{\int_{\Omega}|\nabla u|^{2} d x: u \in V, \int_{\Omega} q(x) u^{2} d x+\int_{\Gamma} q(s) u^{2} d s=1\right\}
$$

then $\Lambda>0$, which is achieved by some $\varphi_{\Lambda} \in V$ with $\varphi_{\Lambda}(x)>0$ a.e. in $\Omega$; see Lemma 1 .

We denote by $c, c_{1}, c_{2}$ universal constants unless specified otherwise. Our main results are as follows.

Theorem 1 Let conditions (S1) to (S3) hold, then:

(i) If $\Lambda>1$, then the problem (1) has no any positive solution in $V$.

(ii) If $\Lambda<1$, then the problem (1) has at least one positive solution in $V$.

(iii) If $\Lambda=1$, then the problem (1) has one positive solution $u(x) \in V$ if and only if there exists a constant $c>0$ such that $u(x)=c \varphi_{\Lambda}(x)$ and $f(x, u)=q(x) u(x)$, $g(x, u)=q(x) u(x)$ a.e. $x \in \Omega$, where $\varphi_{\Lambda}(x)>0$ is the function which achieves $\Lambda$.

Corollary 2 Let conditions (S1) to (S3) with $q(x) \equiv l>0$ hold, then:

(i) If $l<\lambda_{1}$, then the problem (1) has no any positive solution in $V$.

(ii) If $\lambda_{1}<l<+\infty$, then the problem (1) has at least one positive solution in $V$.

(iii) If $l=\lambda_{1}$, then the problem (1) has one positive solution $u(x) \in V$ if and only if there exists a constant $c>0$ such that $u(x)=c \varphi_{1}(x)$ and $f(x, u)=\lambda_{1} u(x), g(x, u)=\lambda_{1} u(x)$ a.e. $x \in \Omega$, where $\varphi_{1}(x)>0$ is the eigenfunction of the $\lambda_{1}$.

Theorem 3 Let conditions (S1) to (S4) with $q(x) \equiv+\infty$ hold, then the problem (1) has at least one positive solution in $V$.

\section{Some lemmas}

We need the following lemmas.

Lemma 1 If $q(x) \in L^{\infty}(\Omega), q(x) \geq 0, q(x) \not \equiv 0$, then $\Lambda>0$ and there exists $\varphi_{\Lambda}(x) \in V$ such that $\Lambda=\int_{\Omega}\left|\nabla \varphi_{\Lambda}\right|^{2} d x$ and $\int_{\Omega} q(x) \varphi_{\Lambda}^{2} d x+\int_{\Gamma} q(s) \varphi_{\Lambda}^{2} d s=1$. Moreover, $\varphi_{\Lambda}(x)>0$ a.e. in $V$.

Proof By the Sobolev embedding function $V \hookrightarrow L^{2}(\Omega)$ and Fatou's lemma, it is easy to know that $\Lambda>0$ and there exists $\varphi_{\Lambda}(x) \in V$, which satisfies $\Lambda$, that is, $\int_{\Omega} q(x) \varphi_{\Lambda}^{2} d x+$ $\int_{\Gamma} q(s) \varphi_{\Lambda}^{2} d s=1$. Furthermore, we assume $\varphi_{\Lambda}(x) \geq 0$, then $\varphi_{\Lambda}(x)$ could replace by $\left|\varphi_{\Lambda}(x)\right|$. By the Strong maximum principle, we know $\varphi_{\Lambda}(x)>0$ a.e. in $V$.

Lemma 2 If conditions (S1) to (S3) hold, then there exists $\beta, \rho>0$ such that $\left.J\right|_{\partial B_{\rho}(0)} \geq \beta$, $\forall u \in V,\|u\|=\rho$.

Proof By condition (S3), there exists $\delta>0, \varepsilon>0$ such that $\frac{f(x, u)}{u} \leq \lambda_{1}-\varepsilon, \frac{g(x, u)}{u} \leq \frac{\gamma f(x, u)}{u} \leq$ $\lambda_{1}-\varepsilon$ as $0<|u| \leq \delta$. Which implies that $F(x, u) \leq \frac{1}{2}\left(\lambda_{1}-\varepsilon\right) u^{2}+c|u|^{y}, G(x, u) \leq \frac{1}{2}\left(\lambda_{1}-\varepsilon\right) u^{2}+$ $c|u|^{z}$. 
By (4) and (5), we obtain

$$
\begin{aligned}
J(u)= & \frac{1}{2}\|\nabla u\|_{L^{2}(\Omega)}^{2}-\int_{\Omega} F(x, u) d x-\int_{\Gamma} G(s, u) d s \\
\geq & \frac{1}{2}\|\nabla u\|_{L^{2}(\Omega)}^{2}+\frac{1}{2}\|\gamma u\|_{L^{2}(\Gamma)}^{2}-\frac{1}{2}\|\gamma u\|_{L^{2}(\Gamma)}^{2}-\frac{1}{2}\left(\lambda_{1}-\varepsilon\right)\|u\|_{L^{2}(\Omega)}^{2} \\
& -c\|u\|_{L^{y}(\Omega)}^{y}-\frac{1}{2}\left(\lambda_{1}-\varepsilon\right)\|u\|_{L^{2}(\Gamma)}^{2}-c\|u\|_{L^{z}(\Gamma)}^{z} \\
\geq & \frac{1}{2}\|u\|^{2}-\frac{1}{2}\left(\lambda_{1}-\varepsilon\right) \frac{1}{\lambda_{1}}\|u\|^{2}-c \gamma_{y}^{y}\|u\|^{y}-\frac{1}{2}\left(\lambda_{1}-\varepsilon+1\right) \frac{1}{\lambda_{1}+1}\|u\|^{2}-c k_{z}^{z}\|u\|^{z} \\
= & {\left[\frac{\varepsilon\left(2 \lambda_{1}+1\right)}{2 \lambda_{1}\left(\lambda_{1}+1\right)}-\frac{1}{2}\right]\|u\|^{2}-c \gamma_{y}^{y}\|u\|^{y}-c k_{z}^{z}\|u\|^{z} . }
\end{aligned}
$$

Hence, $y, z>2$; we take $\varepsilon$ which satisfies $\frac{\varepsilon\left(2 \lambda_{1}+1\right)}{2 \lambda_{1}\left(\lambda_{1}+1\right)}-\frac{1}{2}>0$, that is, $\varepsilon>\frac{\lambda_{1}\left(\lambda_{1}+1\right)}{2 \lambda_{1}+1}$. Then we take a positive constant $\beta$ such that $\left.J\right|_{\partial B_{\rho}(0)} \geq \beta$ as $\|u\|=\rho$, and is small enough.

Lemma 3 If conditions (S1) to (S3) hold, $\Lambda<1, \varphi_{\Lambda}(x)>0$ is defined by Lemma 1, then $J\left(t \varphi_{\Lambda}(x)\right) \rightarrow-\infty$ as $t \rightarrow+\infty$.

Proof If $\Lambda<1, \varphi_{\Lambda}(x)>0$ is defined by Lemma 1, by Fatou's lemma, and (S3), we have

$$
\begin{aligned}
& \lim _{t \rightarrow+\infty} \frac{J\left(t \varphi_{\Lambda}(x)\right)}{t^{2}} \\
&=\frac{1}{2} \int_{\Omega}\left|\nabla \varphi_{\Lambda}(x)\right|^{2} d x-\lim _{t \rightarrow+\infty} \frac{\int_{\Omega} F\left(x, t \varphi_{\Lambda}(x)\right) d x}{t^{2}}-\lim _{t \rightarrow+\infty} \frac{\int_{\Gamma} G\left(s, t \varphi_{\Lambda}(s)\right) d s}{t^{2}} \\
& \leq \frac{1}{2} \int_{\Omega}\left|\nabla \varphi_{\Lambda}(x)\right|^{2} d x-\int_{\Omega} \lim _{t \rightarrow+\infty} \frac{F\left(x, t \varphi_{\Lambda}(x)\right)}{t^{2} \varphi_{\Lambda}^{2}(x)} \varphi_{\Lambda}^{2}(x) d x \\
&-\int_{\Gamma} \lim _{t \rightarrow+\infty} \frac{G\left(s, t \varphi_{\Lambda}(s)\right)}{t^{2} \varphi_{\Lambda}^{2}(s)} \varphi_{\Lambda}^{2}(s) d s \\
&= \frac{1}{2} \int_{\Omega}\left|\nabla \varphi_{\Lambda}(x)\right|^{2} d x-\frac{1}{2} \int_{\Omega} \frac{f\left(x, t \varphi_{\Lambda}(x)\right)}{t \varphi_{\Lambda}(x)} \varphi_{\Lambda}^{2}(x) d x-\frac{1}{2} \int_{\Gamma} \frac{g\left(s, t \varphi_{\Lambda}(s)\right)}{t \varphi_{\Lambda}(s)} \varphi_{\Lambda}^{2}(s) d s \\
&= \frac{1}{2} \int_{\Omega}\left|\nabla \varphi_{\Lambda}(x)\right|^{2} d x-\frac{1}{2}\left[\int_{\Omega} q(x) \varphi_{\Lambda}^{2}(x) d x+\int_{\Gamma} q(s) \varphi_{\Lambda}^{2}(s) d s\right] \\
&= \frac{1}{2 \Lambda}(\Lambda-1) \int_{\Omega}\left|\nabla \varphi_{\Lambda}(x)\right|^{2} d x \\
&< 0 .
\end{aligned}
$$

So, $J\left(t \varphi_{\Lambda}(x)\right) \rightarrow-\infty$ as $t \rightarrow+\infty$.

Lemma 4 Let conditions (S1) and (S2) hold. If a sequence $\left\{u_{n}\right\} \subset V$ satisfies $\left\langle J^{\prime}\left(u_{n}\right), u_{n}\right\rangle \rightarrow$ 0 as $n \rightarrow+\infty$, then there exists a subsequence of $\left\{u_{n}\right\}$, still denoted by $\left\{u_{n}\right\}$ such that $J\left(t u_{n}\right) \leq$ $\frac{1+t^{2}}{2 n}+J\left(u_{n}\right)$ for all $t>0, n \geq 1$.

Proof Since $\left\langle J^{\prime}\left(u_{n}\right), u_{n}\right\rangle \rightarrow 0$ as $n \rightarrow+\infty$, for a subsequence, we may assume that

$$
-\frac{1}{n}<\left\langle J^{\prime}\left(u_{n}\right), u_{n}\right\rangle=\left\|\nabla u_{n}\right\|_{L^{2}(\Omega)}^{2}-\int_{\Omega} f\left(x, u_{n}\right) u_{n} d x-\int_{\Gamma} g\left(s, u_{n}\right) u_{n} d s<\frac{1}{n}, \quad \forall n \geq 1
$$


For any fixed $x \in \Omega$ and $n \geq 1$, set

$$
\psi_{1}(t)=\frac{t^{2}}{2} f\left(x, u_{n}\right) u_{n}-F\left(x, t u_{n}\right), \quad \psi_{2}(t)=\frac{t^{2}}{2} g\left(s, u_{n}\right) u_{n}-G\left(s, t u_{n}\right)
$$

Then (S2) implies that

$$
\begin{aligned}
\psi_{1}^{\prime}(t) & =t f\left(x, u_{n}\right) u_{n}-f\left(x, t u_{n}\right) u_{n} \\
& =t u_{n}\left[f\left(x, u_{n}\right)-\frac{f\left(x, t u_{n}\right)}{t}\right] \\
& = \begin{cases}\geq 0, & 0<t \leq 1 ; \\
\leq 0, & t>1\end{cases}
\end{aligned}
$$

It implies that $\psi_{1}(t) \leq \psi_{1}(1), \forall t>0$. Following the same procedures, we obtain $\psi_{2}(t) \leq$ $\psi_{2}(1), \forall t>0$.

For all $t>0$ and positive integer $n$, by (8), we have

$$
\begin{aligned}
J\left(t u_{n}\right)= & \frac{t^{2}}{2}\left\|\nabla u_{n}\right\|_{L^{2}(\Omega)}^{2}-\int_{\Omega} F\left(x, t u_{n}\right) d x-\int_{\Gamma} G\left(s, t u_{n}\right) d s \\
\leq & \frac{t^{2}}{2}\left[\frac{1}{n}+\int_{\Omega} f\left(x, u_{n}\right) u_{n} d x+\int_{\Gamma} g\left(s, u_{n}\right) u_{n} d s\right] \\
& -\int_{\Omega} F\left(x, t u_{n}\right) d x-\int_{\Gamma} G\left(s, t u_{n}\right) d s \\
\leq & \frac{t^{2}}{2 n}+\int_{\Omega}\left[\frac{1}{2} f\left(x, u_{n}\right) u_{n}-F\left(x, u_{n}\right)\right] d x+\int_{\Gamma}\left[\frac{1}{2} g\left(s, u_{n}\right) u_{n}-G\left(s, u_{n}\right)\right] d s .
\end{aligned}
$$

On the other hand, by (8), one has

$$
\begin{aligned}
J\left(u_{n}\right) & =\frac{1}{2}\left\|\nabla u_{n}\right\|_{L^{2}(\Omega)}^{2}-\int_{\Omega} F\left(x, u_{n}\right) d x-\int_{\Gamma} G\left(s, u_{n}\right) d s \\
& \geq \frac{1}{2}\left[-\frac{1}{n}+\int_{\Omega} f\left(x, u_{n}\right) u_{n} d x+\int_{\Gamma} g\left(s, u_{n}\right) u_{n} d s\right]-\int_{\Omega} F\left(x, u_{n}\right) d x-\int_{\Gamma} G\left(s, u_{n}\right) d s \\
& =-\frac{1}{2 n}+\int_{\Omega}\left[\frac{1}{2} f\left(x, u_{n}\right) u_{n}-F\left(x, u_{n}\right)\right] d x+\int_{\Gamma}\left[\frac{1}{2} g\left(s, u_{n}\right) u_{n}-G\left(s, u_{n}\right)\right] d s
\end{aligned}
$$

One has

$$
\int_{\Omega}\left[\frac{1}{2} f\left(x, u_{n}\right) u_{n}-F\left(x, u_{n}\right)\right] d x+\int_{\Gamma}\left[\frac{1}{2} g\left(s, u_{n}\right) u_{n}-G\left(s, u_{n}\right)\right] d s \leq J\left(u_{n}\right)+\frac{1}{2 n} .
$$

Combining (9) and (10), we have $J\left(t u_{n}\right) \leq \frac{1+t^{2}}{2 n}+J\left(u_{n}\right)$.

Lemma 5 (see [21]) Suppose $E$ is a real Banach space, $J \in C^{1}(E, \mathbb{R})$ satisfies the following geometrical conditions:

(i) $J(0)=0$; there exists $\rho>0$ such that $\left.J\right|_{\partial B_{\rho}(0)} \geq r>0$; 
(ii) There exists $e \in E \backslash \overline{B_{\rho}(0)}$ such that $J(e) \leq 0$. Let $\Gamma_{1}$ be the set of all continuous paths joining 0 and $e$ :

$$
\Gamma_{1}=\{h \in C([0,1], E) \mid h(0)=0, h(1)=e\}
$$

and

$$
c=\inf _{h \in \Gamma_{1}} \max _{t \in[0,1]} J(h(t)) .
$$

Then there exists a sequence $\left\{u_{n}\right\} \subset E$ such that $J\left(u_{n}\right) \rightarrow c \geq \beta$ and $\left(1+\left\|u_{n}\right\|\right) \times$ $\left\|J^{\prime}\left(u_{n}\right)\right\|_{E^{*}} \rightarrow 0$.

\section{Proofs of main results}

Proof of Theorem 1 (i) If $u \in V$ is one positive solution of problem (1), by (3), one has

$$
0=\left\langle J^{\prime}(u), u\right\rangle=\int_{\Omega}|\nabla u|^{2} d x-\int_{\Omega} f(x, u) u d x-\int_{\Gamma} g(s, u) u d s .
$$

That is,

$$
\begin{aligned}
\int_{\Omega}|\nabla u|^{2} d x & =\int_{\Omega} f(x, u) u d x+\int_{\Gamma} g(s, u) u d s \\
& \leq \int_{\Omega} q(x) u^{2} d x+\int_{\Gamma} q(s) u^{2} d s=1 .
\end{aligned}
$$

It implies that $\Lambda \leq 1$. This completes the proof of Theorem 1(i).

(ii) By Lemma 2, there exists $\beta, \rho>0$ such that $\left.J\right|_{\partial B_{\rho}(0)} \geq \beta$ with $\|u\|=\rho$. By Lemma 3, we obtain $J\left(t_{0} \varphi_{\Lambda}(x)\right)<0$ as $t_{0} \rightarrow+\infty$. Define

$$
\begin{aligned}
& \Gamma_{1}=\left\{h \in C([0,1], V) \mid h(0)=0, h(1)=t_{0} \varphi_{\Lambda}(x)\right\}, \\
& c=\inf _{h \in \Gamma_{1}} \max _{t \in[0,1]} J(h(t)),
\end{aligned}
$$

where $\varphi_{\Lambda}(x)>0$ is given by Lemma 1 . Then $c \geq \beta>0$ and by Lemma 3 , there exists $\left\{u_{n}\right\} \subset$ $V$ such that

$$
\begin{aligned}
& J\left(u_{n}\right)=\frac{1}{2}\left\|\nabla u_{n}\right\|_{L^{2}(\Omega)}^{2}-\int_{\Omega} F\left(x, u_{n}\right) d x-\int_{\Gamma} G\left(s, u_{n}\right) d s=c+o(1), \\
& \left(1+\left\|u_{n}\right\|\right)\left\|J^{\prime}\left(u_{n}\right)\right\|_{V^{*}} \rightarrow 0 .
\end{aligned}
$$

(14) implies that

$$
\left\langle J^{\prime}\left(u_{n}\right), u_{n}\right\rangle=\left\|\nabla u_{n}\right\|_{L^{2}(\Omega)}^{2}-\int_{\Omega} f\left(x, u_{n}\right) u_{n} d x-\int_{\Gamma} g\left(s, u_{n}\right) u_{n} d s=o(1) .
$$

Here, in what follows, we use $o(1)$ to denote any quantity which tends to zero as $n \rightarrow+\infty$. 
If $\left\{u_{n}\right\}$ is bounded in $V$, when $\Omega$ is bounded and $f(x, u), g(x, u)$ are subcritical, we can get $\left\{u_{n}\right\}$ has a subsequence strong convergence to a critical value of $J$, and our proof is complete. So, to prove the theorem, we only need show that $\left\{u_{n}\right\}$ is bounded in $V$. Supposing that $\left\{u_{n}\right\}$ is unbounded, that is, $\left\|u_{n}\right\| \rightarrow+\infty$ as $n \rightarrow+\infty$. We order

$$
t_{n}=\frac{2 \sqrt{c}}{\left\|u_{n}\right\|}, \quad w_{n}=t_{n} u_{n}=\frac{2 \sqrt{c} u_{n}}{\left\|u_{n}\right\|} .
$$

Then $\left\{w_{n}\right\}$ is bounded in $V$. By extracting a subsequence, we suppose $w_{n} \rightarrow w$ is a strong convergence in $L^{2}(\Omega), w_{n} \rightarrow w$ is a convergence a.e. $x \in \Omega, w_{n} \rightarrow w$ is a weak convergence in $V$.

We claim that $w \neq 0$. In fact, by (S1) and (S3), we know $\forall x \in \Omega, u_{n} \geq 0$, and there exists $M_{1}, M_{2}>0$ such that $\left|\frac{f\left(x, u_{n}\right)}{u_{n}}\right| \leq M_{1},\left|\frac{g\left(x, u_{n}\right)}{u_{n}}\right| \leq M_{2}$. If $w=0, w_{n} \rightarrow 0$ is a strong convergence in $L^{2}(\Omega)$, and by (15) and (16) we know

$$
\begin{aligned}
4 c & =t_{n}^{2}\left\|u_{n}\right\|^{2}=t_{n}^{2}\left(\left\|\nabla u_{n}\right\|_{L^{2}(\Omega)}^{2}+\left\|\gamma u_{n}\right\|_{L^{2}(\Gamma)}^{2}\right) \\
& =t_{n}^{2} \int_{\Omega} f\left(x, u_{n}\right) u_{n} d x+t_{n}^{2} \int_{\Gamma} g\left(s, u_{n}\right) u_{n} d s+t_{n}^{2}\left\|\gamma u_{n}\right\|_{L^{2}(\Gamma)}^{2}+o(1) \\
& =\int_{\Omega} \frac{f\left(x, u_{n}\right)}{u_{n}} w_{n}^{2} d x+\int_{\Gamma} \frac{g\left(s, u_{n}\right)}{u_{n}} w_{n}^{2} d s+t_{n}^{2}\left\|u_{n}\right\|_{L^{2}(\Gamma)}^{2}+o(1) \\
& \leq M_{1} \int_{\Omega} w_{n}^{2} d x+M_{2} \int_{\Gamma} w_{n}^{2} d s+\left\|w_{n}\right\|_{L^{2}(\Gamma)}^{2}+o(1) \\
& \rightarrow 0 .
\end{aligned}
$$

It is contradiction with $c>0$, so $w \neq 0$.

As follows, we prove $w \neq 0$ satisfies

$$
\int_{\Omega} \nabla \varphi(x) \nabla w(x) d x-\int_{\Omega} q_{1}(x) \varphi(x) w(x) d x-\int_{\Gamma} q_{2}(s) \varphi(s) w(s) d s=0 .
$$

We order

$$
\begin{aligned}
& p_{n}(x)= \begin{cases}f\left(x, u_{n}\right) / u_{n}, & u_{n} \geq 0, x \in \Omega, \\
0, & u_{n}<0, x \in \Omega,\end{cases} \\
& q_{n}(x)= \begin{cases}g\left(x, u_{n}\right) / u_{n}, & u_{n} \geq 0, x \in \Gamma, \\
0, & u_{n}<0, x \in \Gamma .\end{cases}
\end{aligned}
$$

By (S1) and (S3), there exists $M_{3}>0$ such that $0 \leq p_{n}(x) \leq M_{3}, 0 \leq q_{n}(x) \leq M_{3}, \forall x \in \bar{\Omega}$. We select a suitable subsequence and there exists $h_{1}(x) \in L^{2}(\Omega), h_{2}(x) \in L^{2}(\Gamma)$ such that $p_{n}(x) \rightarrow h_{1}(x)$ is a strong convergence in $L^{2}(\Omega), q_{n}(x) \rightarrow h_{2}(x)$ is a strong convergence in $L^{2}(\Gamma)$, and $0 \leq h_{1}(x) \leq M_{3}, 0 \leq h_{2}(x) \leq M_{3}, \forall x \in \bar{\Omega}$.

It follows from $w_{n} \rightarrow w$ is a strong convergence in $L^{2}(\Omega)$ that

$$
\begin{aligned}
& \int_{\Omega} p_{n}(x) w_{n}(x) \varphi(x) d x=\int_{\Omega} p_{n}(x) w_{n}^{+}(x) \varphi(x) d x \rightarrow \int_{\Omega} h_{1}(x) w^{+}(x) \varphi(x) d x, \\
& \int_{\Gamma} q_{n}(s) w_{n}(s) \varphi(s) d s=\int_{\Gamma} q_{n}(s) w_{n}^{+}(s) \varphi(s) d s \rightarrow \int_{\Gamma} h_{2}(s) w^{+}(s) \varphi(s) d s .
\end{aligned}
$$


Hence, $\left\{p_{n}(x) w_{n}(x)\right\}$ is bounded in $L^{2}(\Omega), p_{n}(x) w_{n}(x) \rightarrow h_{1}(x) w^{+}(x)$ in $L^{2}(\Omega) ;\left\{q_{n}(x) w_{n}(x)\right\}$ is bounded in $L^{2}(\Gamma), q_{n}(x) w_{n}(x) \rightarrow h_{2}(x) w^{+}(x)$ in $L^{2}(\Gamma)$.

By (16), we have

$$
\begin{aligned}
& \left|\int_{\Omega} \nabla w_{n}(x) \nabla \varphi(x) d x-\int_{\Omega} p_{n}(x) w_{n}(x) \varphi(x) d x-\int_{\Gamma} q_{n}(s) w_{n}(s) \varphi(s) d s\right| \\
& \quad=\left|\int_{\Omega} \nabla\left(t_{n} u_{n}(x)\right) \nabla \varphi(x) d x-\int_{\Omega} p_{n}(x) t_{n} u_{n}(x) \varphi(x) d x-\int_{\Gamma} q_{n}(s) t_{n} u_{n}(s) \varphi(s) d s\right| \\
& \quad=\frac{2 \sqrt{c}}{\left\|u_{n}\right\|}\left|\int_{\Omega} \nabla u_{n}(x) \nabla \varphi(x) d x-\int_{\Omega} p_{n}(x) u_{n}(x) \varphi(x) d x-\int_{\Gamma} q_{n}(s) u_{n}(s) \varphi(s) d s\right| \\
& \quad \rightarrow 0 .
\end{aligned}
$$

Since $w_{n} \rightarrow w$ is a weak convergence in $V$, we obtain

$$
\int_{\Omega} \nabla \varphi(x) \nabla w(x) d x-\int_{\Omega} h_{1}(x) \varphi(x) w^{+}(x) d x-\int_{\Gamma} h_{2}(s) \varphi(s) w^{+}(s) d s=0, \quad \varphi \in V .
$$

We order $\varphi=w^{-}$; this yields $\left\|w^{-}\right\|^{2}=0$, so $w=w^{+} \geq 0$. By the Strong maximum principle, we know $w>0$ a.e. in $\Omega$, so $u_{n} \rightarrow \infty$ a.e. in $\Omega$. Combining (S3) and (3), we obtain

$$
\int_{\Omega} \nabla \varphi(x) \nabla w(x) d x-\int_{\Omega} q(x) \varphi(x) w(x) d x-\int_{\Gamma} q(s) \varphi(s) w(s) d s=0, \quad \forall \varphi \in V .
$$

This is a contradiction with $\Lambda<1$. This completes the proof of Theorem 1(ii).

(iii) If $\Lambda=1$, by Lemma 1 , there exists some $\varphi_{\Lambda}(x)>0$, such that

$$
\int_{\Omega} \nabla v(x) \nabla \varphi_{\Lambda}(x) d x=\int_{\Omega} q(x) v(x) \varphi_{\Lambda}(x) d x+\int_{\Gamma} q(s) v(s) \varphi_{\Lambda}(s) d s .
$$

If $u$ is a positive solution of (1), for the above $\varphi_{\Lambda}(x)$, we have

$$
\int_{\Omega} \nabla u(x) \nabla \varphi_{\Lambda}(x) d x=\int_{\Omega} f(x, u(x)) \varphi_{\Lambda}(x) d x+\int_{\Gamma} g(s, u(s)) \varphi_{\Lambda}(s) d s .
$$

We order $v=u$ in (17), and it follows from (18) that

$$
\begin{aligned}
\int_{\Omega} \nabla u(x) \nabla \varphi_{\Lambda}(x) d x & =\int_{\Omega} q(x) u(x) \varphi_{\Lambda}(x) d x+\int_{\Gamma} q(s) u(s) \varphi_{\Lambda}(s) d s \\
& =\int_{\Omega} f(x, u(x)) \varphi_{\Lambda}(x) d x+\int_{\Gamma} g(s, u(s)) \varphi_{\Lambda}(s) d s \\
& \leq \int_{\Omega} q(x) u(x) \varphi_{\Lambda}(x) d x+\int_{\Gamma} q(s) u(s) \varphi_{\Lambda}(s) d s
\end{aligned}
$$

which implies that $\int_{\Omega}(f(x, u)-q(x) u(x)) \varphi_{\Lambda}(x) d x+\int_{\Gamma}(g(s, u)-q(s) u(s)) \varphi_{\Lambda}(s) d s=0$.

When $\varphi_{\Lambda}(x)>0$ a.e. in $\Omega$, combining (S2), (S3), and (3), we obtain

$$
f(x, u) \leq q(x) u(x), \quad g(x, u) \leq q(x) u(x)
$$

Then we must have $f(x, u)=q(x) u(x), g(x, u)=q(x) u(x)$ a.e. in $\Omega, u(x)>0$ also achieves $\Lambda(=1)$. When $u=c \varphi_{\Lambda}, c>0$, we have $\int_{\Omega}\left|\nabla \varphi_{\Lambda}\right|^{2} d x=\int_{\Omega} q(x) \varphi_{\Lambda}^{2} d x+\int_{\Gamma} q(s) \varphi_{\Lambda}^{2} d s$, which achieves $\Lambda$. 
On the other hand, if for some $c>0, u(x)=c \varphi_{\Lambda}(x)$ and $f\left(x, c \varphi_{\Lambda}(x)\right)=c q(x) \varphi_{\Lambda}(x), g(x, u)=$ $c q(x) \varphi_{\Lambda}(x)$ a.e. $x \in \Omega$, since $c \varphi_{\Lambda}(x)$ also achieves $\Lambda$. This means $u(x)=c \varphi_{\Lambda}(x)$ is a solution of problem (1) as $\Lambda=1$. This completes the proof of Theorem 1(iii).

Proof of Corollary 2 Note that when $q(x) \equiv l$, then $\Lambda=\frac{\lambda_{1}}{l}$. The conclusion follows from Theorem 1.

Proof of Theorem 3 When $q(x) \equiv+\infty$, we can replace $\varphi_{\Lambda}$ by $\varphi_{1}$ in (11) and define $c$ as in (12), then following the same procedures as in the proof of Theorem 1(ii), we need to show only that $\left\{u_{n}\right\}$ is bounded in $V$. For this purpose, let $\left\{w_{n}\right\}$ be defined as in (16). If $\left\{w_{n}\right\}$ is bounded in $V$, we know $w_{n} \rightarrow w$ is a strong convergence in $L^{2}(\Omega), w_{n} \rightarrow w$ is convergence a.e. $x \in \Omega, w_{n} \rightarrow w$ is a weak convergence in $V$, and $w \in V$.

If $\left\|u_{n}\right\| \rightarrow+\infty$, then $t_{n} \rightarrow 0$ and $w(x) \equiv 0$. We set $\Omega_{1}=\{x \in \Omega: w(x)=0\}, \Omega_{2}=\{x \in \Omega$ : $w(x) \neq 0\}$. Obviously, by (16), $\left|u_{n}\right| \rightarrow+\infty$ a.e. in $\Omega_{2}$. When $q(x) \equiv+\infty$ in (S3), there exists $K_{1}, K_{2}>0$ and $n$ large enough we have $\left|\frac{f\left(x, u_{n}\right)}{u_{n}}\right| \geq K_{1},\left|\frac{g\left(x, u_{n}\right)}{u_{n}}\right| \geq K_{2}$ uniformly in $x \in \Omega_{2}$. Hence, by (15) and (16), we obtain

$$
\begin{aligned}
4 c & =\lim _{n \rightarrow+\infty} t_{n}^{2}\left\|u_{n}\right\|^{2} \\
& =\lim _{n \rightarrow+\infty} t_{n}^{2}\left(\left\|\nabla u_{n}\right\|_{L^{2}(\Omega)}^{2}+\left\|\gamma u_{n}\right\|_{L^{2}(\Gamma)}^{2}\right) \\
& =\lim _{n \rightarrow+\infty} t_{n}^{2}\left(\int_{\Omega} f\left(x, u_{n}\right) u_{n} d x+\int_{\Gamma} g\left(s, u_{n}\right) u_{n} d s+\left\|\gamma u_{n}\right\|_{L^{2}(\Gamma)}^{2}\right) \\
& =\lim _{n \rightarrow+\infty}\left(\int_{\Omega} \frac{f\left(x, u_{n}\right)}{u_{n}} w_{n}^{2} d x+\int_{\Gamma} \frac{g\left(s, u_{n}\right)}{u_{n}} w_{n}^{2} d s+t_{n}^{2}\left\|\gamma u_{n}\right\|_{L^{2}(\Gamma)}^{2}\right) \\
& \geq K_{1} \int_{\Omega} w^{2} d x+K_{2} \int_{\Gamma} w^{2} d s+\|w\|_{L^{2}(\Gamma)}^{2} .
\end{aligned}
$$

Noticing that $w(x) \neq 0$ in $\Omega_{2}$ and $K_{1}, K_{2}$ can be chosen large enough, so $m \Omega_{2} \equiv 0$ and then $w(x) \equiv 0$ in $\Omega$.

Then we know $\lim _{n \rightarrow+\infty} \int_{\Omega} F\left(x, w_{n}\right) d x+\lim _{n \rightarrow+\infty} \int_{\Gamma} G\left(s, w_{n}\right) d s=0$, and consequently,

$$
\begin{aligned}
J\left(w_{n}\right) & =\frac{1}{2}\left\|\nabla w_{n}\right\|_{L^{2}(\Omega)}^{2}+o(1) \\
& =\frac{1}{2}\left\|w_{n}\right\|^{2}-\frac{1}{2}\left\|w_{n}\right\|_{L^{2}(\Gamma)}^{2}+o(1) \\
& \geq \frac{1}{2}\left(1-\frac{1}{\lambda_{1}+1}\right)\left\|w_{n}\right\|^{2}+o(1) \\
& =2 c\left(1-\frac{1}{\lambda_{1}+1}\right)+o(1)
\end{aligned}
$$

By $\left\|u_{n}\right\| \rightarrow+\infty, t_{n} \rightarrow 0$ as $n \rightarrow+\infty$, then it follows Lemma 4 and (13), we obtain

$$
J\left(w_{n}\right)=J\left(t_{n} u_{n}\right) \leq \frac{1+t_{n}^{2}}{2 n} \leq c .
$$

Obviously, (19) and (20) are contradictory. So $\left\{u_{n}\right\}$ is bounded in $V$. This completes the proof of Theorem 3. 


\section{Example}

In this section, we give two examples on $f(x, u)$ : One satisfies (S1) to (S3) with $q(x) \equiv+\infty$, but does not satisfy the (AR) condition; the other illustrates how the assumptions on the boundary are not trivial and compatible with the inner assumptions in $\Omega$.

Example 1 Set:

$$
f(x, t)= \begin{cases}0, & t \leq 0 \\ t \ln (1+t), & t>0\end{cases}
$$

Then it is easy to verify that $f(x, t)$ satisfies (S1) to (S3) with $p(x)=0$ as $t \rightarrow 0$ and $q(x)=$ $+\infty$ as $t \rightarrow+\infty$. In addition,

$$
F(x, t)=\frac{1}{2} t^{2} \ln (1+t)-\frac{1}{4} t^{2}+\frac{1}{2} t-\frac{1}{2} \ln (1+t) .
$$

So, for some $\mu>2, \mu F(x, t)=t^{2} \ln (1+t)\left(\frac{\mu}{2}-\frac{\mu}{4 \ln (1+t)}+\frac{\mu}{2 \ln (1+t)}-\frac{\mu}{2 t^{2}}\right)>t^{2} \ln (1+t)$, for all $t$ large.

This means $f(x, t)$ does not satisfy the (AR) condition.

Example 2 Consider the following problem:

$$
\left\{\begin{array}{l}
-u^{\prime \prime}(x)=\alpha u(x), \quad 0<x<l, \\
u(0)=0 \\
u^{\prime}(l)=\alpha u(l)
\end{array}\right.
$$

where $\alpha>0$ is a constant. It is obvious that $g=\gamma f$ as $f(x, u)=\alpha u(x)$. Problem (21) is a case of (1); we can obtain the nontrivial solution: $u(x)=\widetilde{C} \sin \sqrt{\alpha} x, \widetilde{C} \neq 0$.

\section{Competing interests}

The author declares that he has no competing interests.

\section{Author's contributions}

Li G carried out all studies in this article.

\section{Acknowledgements}

The author would like to thank the referees for carefully reading this article and making valuable comments and suggestions.

Received: 19 January 2012 Accepted: 6 August 2012 Published: 16 August 2012

\section{References}

1. Liu, H, Su, N: Well-posedness for a class of mixed problem of wave equations. Nonlinear Anal. 71, 17-27 (2009). doi:10.1016/j.na.2008.10.027

2. Landesman, EM, Lazer, AC: Nonlinear perturbations of linear elliptic boundary value problems at resonance. J. Math. Mech. 19, 609-623 (1970)

3. Ahmad, S, Lazer, AC, Paul, JL: Elementary critical point theory and perturbations of elliptic boundary value problems at resonance. Indiana Univ. Math. J. 25, 933-944 (1976)

4. Berestycki, H, De Figueiredo, DG: Double resonance in semilinear elliptic problems. Commun. Partial Differ. Equ. 6 , 91-120 (1981)

5. Costa, DG, Oliveira, AS: Existence of solution for a class of semilinear elliptic problems at double resonance. Bol. Soc. Bras. Mat. 19, 21-37 (1988)

6. Omari, P, Zanolin, F: Resonance at two consecutive eigenvalues for semilinear elliptic equations. Ann. Mat. Pura Appl. 163, 181-198 (1993) 
7. Binding, PA, Drábek, P, Huang, YX: Existence of multiple solutions of critical quasilinear elliptic Neumann problems. Nonlinear Anal. 42, 613-629 (2000). doi:10.1016/S0362-546X(99)00118-2

8. Li, G, Zhou, HS: Asymptotically linear Dirichlet problem for the $p$-Laplacian. Nonlinear Anal. 43, 1043-1055 (2001). doi:10.1016/S0362-546X(99)00243-6

9. Escobar, JF: A comparison theorem for the first non-zero Steklov eigenvalue. J. Funct. Anal. 178, 143-155 (2000) doi:10.1006/jfan.2000.3662

10. Kaur, BS, Sreenadh, K: Multiple positive solutions for a singular elliptic equation with Neumann boundary condition in two dimensions. Electron. J. Differ. Equ. 43, 1-13 (2009)

11. Ahmad, S: Multiple nontrivial solutions of resonant and nonresonant asymptoticaly problems. Proc. Am. Math. Soc. 96, 405-409 (1986)

12. Chang, KC: Infinite Dimensional Morse Theory and Multiple Solutions Problems. Birkhäuser, Boston (1993)

13. Hirano, N, Nishimura, T: Multiplicity results for semilinear elliptic problems at resonance and with jumping nonlinearities. J. Math. Anal. Appl. 180, 566-586 (1993). doi:10.1006/jmaa.1993.1417

14. Landesman, E, Robinson, S, Rumbos, A: Multiple solution of semilinear elliptic problem at resonance. Nonlinear Anal. 24, 1049-1059 (1995). doi:10.1016/0362-546X(94)00107-S

15. Liu, SQ, Tang, CL, Wu, XP: Multiplicity of nontrivial solutions of semilinear elliptic equations. J. Math. Anal. Appl. 249, 289-299 (2000). doi:10.1006/jmaa.2000.6704

16. Li, S, Willem, M: Multiple solution for asymptotically linear boundary value problems in which the nonlinearity crosses at least one eigenvalue. Nonlinear Differ. Equ. Appl. 5, 479-490 (1998)

17. Mawhin, J, Willem, M: Critical Point Theory and Hamiltonien Systems. Springer, New York (1989)

18. Lions, JL, Magenes, E: Non-Homogeneous Boundary Value Problems and Applications. Springer, Berlin (1972)

19. Zhou, H: An application of a mountain pass theorem. Acta Math. Sin. Engl. Ser. 18(1), 27-36 (2002)

20. Cheng, BT, Wu, X: Existence results of positive solutions of Kirchhoff type problems. Nonlinear Anal. 71, 4883-4892 (2009). doi:10.1016/j.na.2009.03.065

21. Ambrosetti, A, Rabinowitz, PH: Dual variational methods in critical points theory and applications. J. Funct. Anal. 14 349-381 (1973). doi:10.1016/0022-1236(73)90051-7

doi:10.1186/1687-2770-2012-91

Cite this article as: Li: Existence of positive solutions of elliptic mixed boundary value problem. Boundary Value Problems 2012 2012:91.

\section{Submit your manuscript to a SpringerOpen ${ }^{\circ}$ journal and benefit from:}

- Convenient online submission

- Rigorous peer review

- Immediate publication on acceptance

- Open access: articles freely available online

- High visibility within the field

- Retaining the copyright to your article 